\title{
FRAMEWORK FOR A MULTI-LEVEL EVOLUTIONARY ALGORITHM FOR CONSTRUCTION OPTIMIZATION
}

\author{
Mohamed Abdel-Raheem and Ahmed Khalafallah \\ Department of Civil, Environmental and Construction Engineering. \\ University of Central Florida \\ 4000 Central Florida Blvd. Orlando, Florida, 32816, USA \\ Email: abdelrah@mail.ucf.edu \\ khalafal@mail.ucf.edu
}

\section{KEYWORDS}

Evolutionary

Construction.

Algorithms,

Optimization,

\begin{abstract}
In large-scale non-linear construction optimization problems, the capability of an algorithm to find the optimal solution is usually limited by the inability to evaluate the effect of change in the value of each decision variable on the overall outcome of the objective function. Current optimization algorithms evaluate the quality of generated solutions based only on the value of fitness/objective function. As such, these algorithms are limited in their ability to robustly reach optimal solutions. This paper presents a framework for an innovative evolutionary algorithm that mimics the behavior of electrons moving through electric circuit branches with the least resistance. In the proposed algorithm, solutions are evaluated on two levels: a global level against the objective function; and a local level by evaluating the potential of the generated value for each decision variable. This paper presents (1) the philosophy behind this work; (2) the concept adopted in developing the algorithm; and (3) the basic steps of the algorithm. The new algorithm is expected to enhance the optimization of complex large-scale optimization problems.
\end{abstract}

\section{INTRODUCTION}

Optimization plays an important role in enhancing the efficiency and ensuring the economic feasibility of various applied systems in the construction industry. Throughout the years, several optimization techniques, algorithms, and models were developed to deal with a multitude of problems within the industry. In the past, simple mathematical techniques were used to solve many simple optimization problems. With the technological advancement of computing systems and the need for powerful optimization techniques, several sophisticated optimization systems were developed to solve many complex problems that were hard to solve in a polynomial time frame (NP-hard). In 1975, John Holland developed genetic algorithms (GAs) simulating the Darwinian principle of evolution and the survival of the fittest (Goldberg 1989). Several other algorithms followed, modifying the original approach, in order to overcome the limitations demonstrated in the inability to reach optimum/near optimum solutions in reasonable time. In 1989, Pablo Moscato developed the memetic algorithm (MA) based also on the Darwinian principle of evolution, but his main contribution relied in adopting Dawkins' concept of a meme. Other EAs were also developed simulating various natural phenomena of biological evolution or social behavior of individuals. Example of these EAs are: particle swarm optimization (PSO), ant colony optimization (ACO), shuffled frog leaping (SFL), harmony search (HS) and others (Elbeltagi et al. 2005).

In the past decade, the application of evolutionary algorithms (EAs) in construction served as a quite acceptable alternative technique for solving complex optimization problems. Examples of EA applications appeared in various engineering disciplines (Feng et al.1997; Leu and Yang 1999; Haidar et al. 1999; Hegazy and Ersahin 2001; Hegazy and Wassed 2001; Lu 2002; Maier et al. 2003; Shyu et al. 2004; Solimanpur et al. 2004; Aguilar 2004; Christodoulou 2005; Elazouni and Metwally 2005; Elbeltagi et al. 2005; El-Rayes and Kandil 2005; Zehng et al. 2005; Khalafallah and El-Rayes 2006).

Most EAs are directed towards reaching optimality through the simulation of the behavior of certain species in certain ecological systems. The diversity in types of creatures that have evolved over the centuries makes the number and types of EAs simulating their behavior unlimited (Wu and Banzhaf 1998). In EAs, a pool of solutions (population) is created, where each individual in the population is modeled as an array. The merit of each array is evaluated with respect to an objective function throughout several cycles of evolution. 


\section{PROBLEM DEFINITION AND STUDY OBJECTIVES}

The developed EAs are considered in many cases computationally inefficient, especially in optimizing large-scale complex construction systems. In many of these complex optimization problems, it could take several days of parallel computing in order to reach a near optimal solution. This limitation could be attributed to two main factors.

First, EAs deal with candidate solutions indifferently and do not consider the potential of each individual solution. For instance, GAs simulate the behavior of human beings through its search for the best genes in a population of chromosomes representing a group of individuals. All genes constituting a chromosome receive same appreciation, when the fact is there are good and bad genes within the same chromosome. Although the processes of crossover and mutation may help in promoting some of the promising individuals and/or genes, chances of reaching the chromosome with the optimal solution is very limited. Another example is the individual ant in $\mathrm{ACO}$, where each ant represents a solution string whose values receive the same pheromone reward regardless of the individual quality of the values in the solution string.

Second, current EAs strive to simulate the behavior of change without paying much attention to the change in the surrounding environment. Most species adapt to the changes in the ecological system surrounding them; and that is the main reason of their survival. They adapt by changing their behavior, while most EAs adopt the common behavior that is not necessarily guaranteed to be the best. This greatly affects the efficiency of the algorithm. For example, Dorigo's ACO is based on simulating the foraging behavior of ants and their ability to select the shortest paths between nests and sources of food. Deneubourg and collegues in 1989 and 1990 investigated the foraging behavior of ants through an experiment in which they utilized a double bridge to connect a source of food with a nest of ants of the Argentine type. They ran three experiments; in each one, they changed the lengths of the bridges. In one of the three experiments, the ants did not choose the shortest path (Dorigo, and Stutzle, 2004).

To overcome the shortcomings of other EAs, two points were taken in consideration. First was the quest for a natural phenomenon whose individuals' behavior is always consistent. Second, it was essential to find a way to evaluate the performance of the each entity in the system independently.
This paper presents a framework for a new innovative multi-level evolutionary algorithm under development - that tries to overcome the aforementioned limitations. The proposed algorithm is designed to mimic the behavior of electrons in a multi-branch electric circuit, where the majority of electrons join the branch with the least resistance. The following sections illustrate the methodology adopted for this study, the proposed multi-level evolutionary algorithm, and the planned future efforts to overcome the current algorithm limitations.

\section{RESEARCH METHODOLOGY}

The electric current is induced by the flow of electrons in a designated medium. The intensity of current induced is based on the resistance of the medium to the flow of electrons through it. Therefore, changing the medium's resistance will change the intensity of the current; i.e. number of electrons passing through it.

In the proposed algorithm, candidate solutions for the objective function are generated randomly. Each solution represents a wire with an unknown resistance. Each value in the solution string represents a segment in the wire of different material and different resistance connected in series as shown in Figure 1. Therefore, there is a global resistance for the whole wire (solution string) and a local resistance for each value comprising the solution.

The individuals of the population of solution strings (wires) are connected in parallel representing an electric circuit as shown in Figure 2. The electrons flow in the circuit due to a potential difference in a source whose voltage is selected based on the type of the problem. Each wire is evaluated globally with respect to all the other wires, and each segment in the wire is compared to other local segments within the same wire. The global and internal individual resistances are then computed for each wire and its segments, as described in more details in the following section. The whole circuit is then demolished, and a new circuit is constructed using the best wires identified in the previous cycle. The process is iterative till the wire with the best conductivity is created (i.e. optimum solution).

The basic difference between the proposed EA and other EAs relies in the evolution process. In traditional EAs, the species (solutions population) evolve and are ranked with respect to an objective function to tell how good they are until they reach superiority (optimum value). 


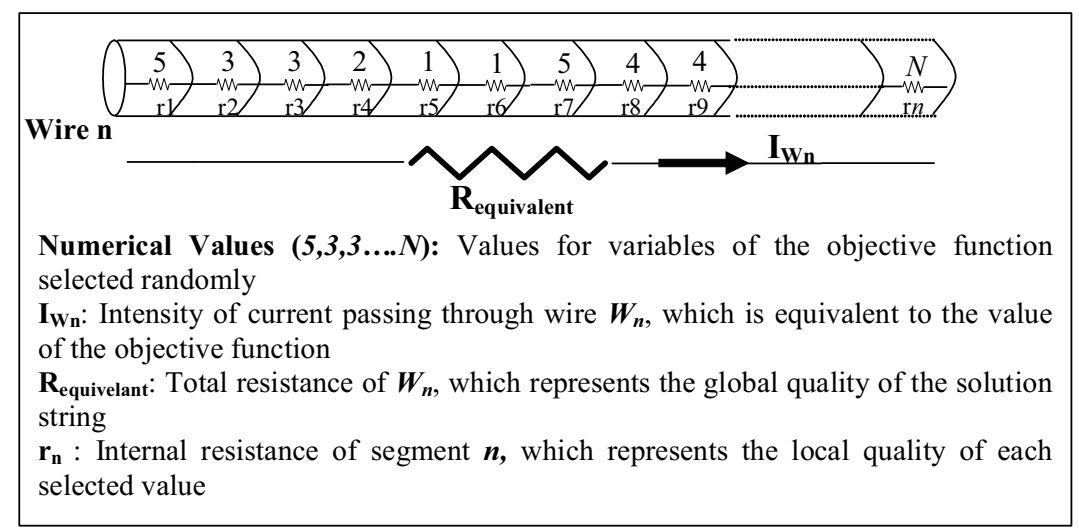

Figure 1: Schematic Diagram of Solution Strings (wires)

On the other hand, in the new EA the electrons do not evolve, but their surrounding environment (electric circuit) does. The electrons are merely identical agents with consistent behavior; they select the path with the least resistivity and go through the electric circuit, telling how good the conditions are.

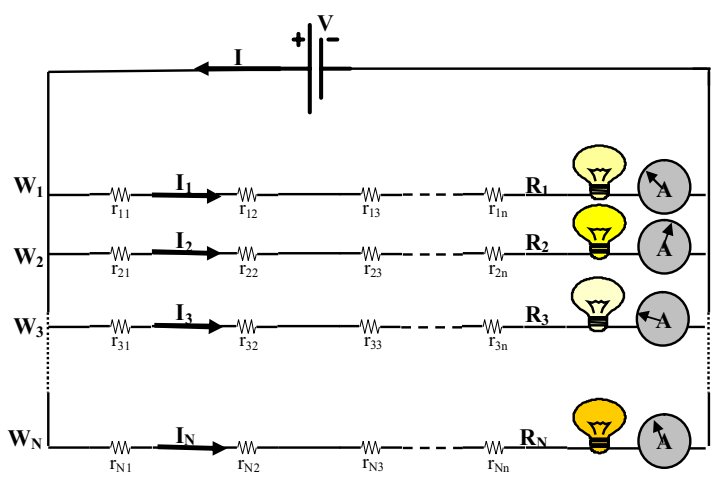

Figure 2: Solution Population Represented as an Electric Circuit

\section{MULTI-LEVEL EVOLUTIONARY ALGORITHM FOR OPTIMIZATION}

The proposed algorithm is iterative where a number of wires (solution strings) are created, tested, and readjusted. The basic steps of the algorithm are as follows:

1. Construction of trial solutions: This is done by fabricating a number of wires $(N)$ composed of $n$ segments of different material connected in serious. Each segment is randomly enumerated to represent a resistance value $r_{n}$ in the solution space, as shown in Figure 1.

2. Construction of the electric circuit: Once all wires are fabricated, they are connected to an electric source of voltage $V$. The value of $V$ is selected based on the type of problem, as shown in Figure 2.
3. Calculation of the current intensity $I$ : For each wire, the intensity of current $I_{N}$ passing through it is calculated. This is achieved by substituting the randomly selected value of each wire in the objective function.

4. Determining resistance of wires $\left(R_{N}\right)$ : The global resistance of each wire is calculated using Ohm's Law: $R_{N}=V / I_{N}$.

5. Quality of wires: This is determined by the relative importance $Z$ of $W_{N}$ compared to other wires in the solution space. The relative importance is based on the current intensity $I_{N}$ according to equation (1).

$$
\text { Relative Importance }(\boldsymbol{Z})=\frac{\mathrm{I}_{\mathrm{N}}}{\sum_{1}^{\mathrm{N}} \mathrm{I}_{\mathrm{N}}}
$$

6. Quality of selected values: The quality of each value in $W_{n}$ is determined by calculating its internal resistance $r_{n}$. At first, it is assumed that all resistances $r_{n}$ for segments $n$ appear identical since there is no prior information about how good the solution is. Therefore the resistances are calculated according to equation (2).

$r_{n v}=\frac{R_{N}}{n}$

In order to determine the actual resistance of each value, a sensitivity analysis is conducted by substituting the value $v$ of each segment in a sample $S$ of the wire population. The change $\Delta r$ is then recorded and the resistance is calculated according to equation (3).

$$
r_{\text {mod ified }}=r_{n v}-\sum_{1}^{S}\left(\Delta r_{n v} \times \mathrm{Z}_{S}\right)
$$

Where $r_{n \mathrm{v}}=$ internal resistance of selected value $v$ in the original wire $W_{n} ; \Delta r_{n v}=$ difference between the original resistance and the new 
resistance after substituting the value $v$ in $W_{s} ; S=$ sample size; $Z_{S}=$ relative importance of the wire selected.

The calculated resistances $r_{n}$ for different values $v_{n}$ of the same wire $W_{n}$ are then normalized with respect to the global resistance $R_{n}$. This guarantees that there is no violation to Kirchhoff's rule.

The sample size $(S)$ can be determined using Cochran's equation (4).

$\eta_{0}=\frac{(t)^{2} *(P)(q)}{(d)^{2}}$

Where: $\eta_{0}=$ sample size; $t=$ value selected for a designated alpha level; $d=$ acceptable margin of error; and $(p)(q)=$ variance in $I_{N}$.

7. Updating resistance $\left(r_{n}\right)$ of selected values: the resistance $r_{n}$ of selected values $v$ is updated according to equation (5):

$r_{\mathrm{v} \text { updated }}=\operatorname{Min}\left[\mathrm{r}_{\mathrm{v}}(\mathrm{m}-1), \mathrm{r}_{\mathrm{v}}(m)\right]+\frac{\operatorname{Min}\left[\mathrm{r}_{\mathrm{v}}(\mathrm{m}-1), \mathrm{r}_{\mathrm{v}}(m)\right]}{\operatorname{Max}\left[\left(\mathrm{r}_{\mathrm{v}}(\mathrm{m}-1), \mathrm{r}_{\mathrm{v}}(m)\right]\right.}$

Where $r_{\text {vupdated }}=$ updated resistance for value $v$; $r_{v}(m-1)=$ resistance of $v$ at previous iteration $(m-$ 1); $r_{v}(m)=$ resistance of $v$ at current iteration (m).

8. Selection of values: The selection process of values is based on the individual resistance associated with each value, which is updated after each iteration until the best conductor is created. The probability of selecting values for each parameter in the objective function is calculated according to equation (6).

$$
P_{v n}\left(w_{N}, m\right)=\frac{1 / r_{v n}}{\sum_{v} 1 / r_{v n}}
$$

Where $\mathrm{P}_{\mathrm{vn}}=$ probability that value $v$ is selected for segment $n$ in wire $W_{N}$ at iteration $m ; \mathrm{r}_{\mathrm{vn}}=$ resistance of value $v$ selected for segment $n$.

It is worth mentioning that this method of selecting values guarantees the deployment of all values in the solution space at least once. This is due to the fact that the initial resistance $r_{n}$ of all values at iteration $(\mathrm{m}=0)$ appear to be identical and have a value of 1 .

The flow chart for the proposed algorithm is shown in Figure 3.

\section{OBSERVATIONS AND FUTURE RESEARCH}

During the development of the algorithm, several hardships were encountered. The main one relied in finding a suitable way to evaluate each value in the solution string independently.

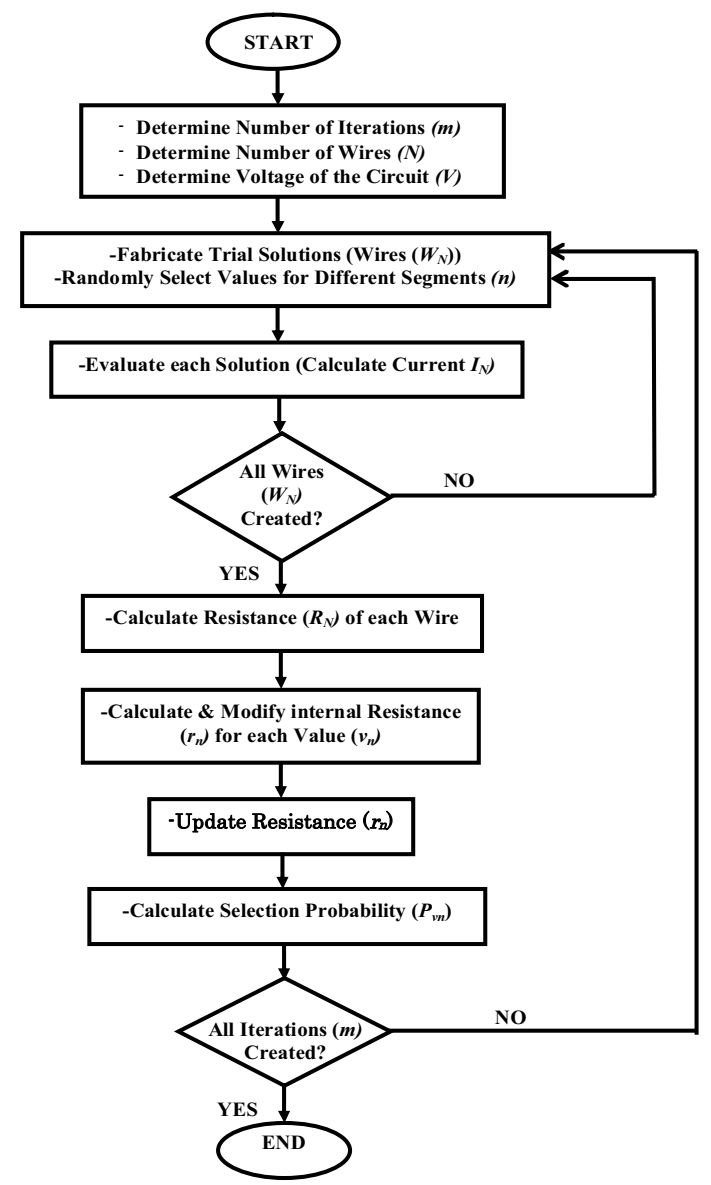

Figure 3: Flowchart of the Proposed Algorithm

As mentioned earlier, each solution string is evaluated globally and locally. The basic idea for evaluating the performances of different values of variables of the objective function revolved around finding a suitable method to calculate the internal resistance of the wire segments. Several attempts were made to come up with a sound way to evaluate each value independently, i.e. calculate local resistance $r_{n}$.

The coefficients of the objective function variables were investigated to serve as a suitable measure for the performance of different values. In optimization, the coefficients of the objective function play a very important role in determining the optimal solution. For instance, in linear programming, changing the coefficient of the objective function changes the optimal solution. Consequently, the variable with the highest coefficient contributes the most in maximization problems (Winston, 1993). Hence, the basic idea was to initially assume that the local resistance $r_{n}$ is a proportion of the global $R_{n}$ with a value of $\left[\left(C_{n} / \Sigma C_{n}\right) \times R_{n}\right]$, where $C_{n}$ is the coefficients of variable $n$. However, this practice is not practical 
for all optimization problems. This is due to the fact that in most cases the solution string does not carry real values of the objective function variables. For example, consider the case study appeared in (Elbeltagi et al., 2005) for the time-cost tradeoff problem. The solution strings carried indices referring to various construction methods of different times and costs. This means that each single value is meaningless if plugged directly in the objective function.

A special attention was given to the signs of the coefficients of the objective function, and the type of connection between the wire segments. The segments of the same wire can be connected in series or parallel based on the sign of the coefficients. Consider the following hypothetical trivial objective function: [Maximize: 7a-3b+4c]. Since it is a maximization problem, the values for variable $a$ and $c$ should be as high as possible, and as low as possible for variable $b$. The ideal way to model this function, is to have the wire segments for variables $a$ and $c$ connected in series, and the segment for variable $b$ connected to them in parallel as shown in Figure 4. However, in most cases and as mentioned earlier, the values of the solution strings do not relate directly to the variable of the objective function. The assumption used in this algorithm is that all segments of the same wire are connected in series. That assumption makes sense for two reasons. First, the series connection would represent the general case for all problem domains. Second, in real life, two points on an electrical circuit could be connected by two or more different wire segments joined in series rather than in parallel.

One of the basic advantages of this algorithm is its ability to evaluate each value of the solution string independently. The concept of the internal resistance of different segments within the same wire was found to be very convenient. This is due to the fact that the value of the internal resistance $r_{n}$ is independent of the value of the objective function. In other words, the internal resistance is merely a measure of how good each value is. This guarantees no violation for Ohm's and Kirchhoff's rules as the only changing value is $I_{N}$.

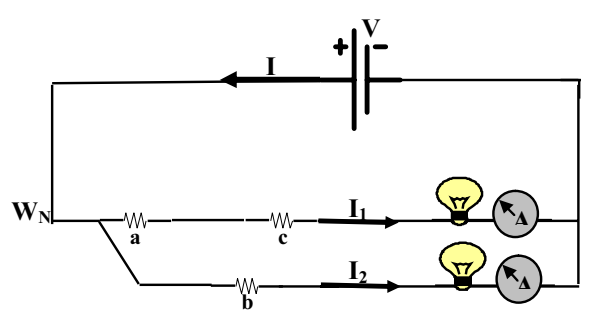

Figure 4. Alternative Representation of the Solution String Based on the Signs of the Coefficients
A very interesting observation that is currently under investigation is the change in the potential of individual values for reaching optimal solution. Consider the electric circuit shown in Figure 5. The two wires are identical except for resistance $r_{l}$. Table 1 summarizes the values of $I, V$, and $R$.

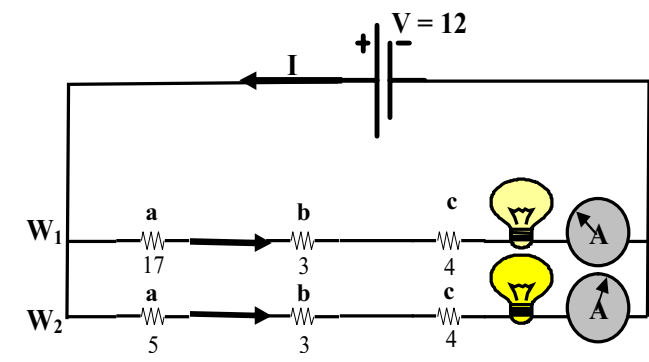

Figure 5. Electric Circuit Representing a Hypothetical Optimization Problem

It can be noticed that when the value of resistance $r_{l}$ changed in the second wire, the value of $I$ across the wire was doubled, and the potential difference across the nodes of resistance $b$, and $c$ was doubled though their resistance values did not change.

Table 1. Summary of Values of I, V, and R of the Circuit in Figure 5

\begin{tabular}{|l|c|c|}
\hline Wire & $\mathbf{1}$ & $\mathbf{2}$ \\
\hline $\mathbf{R}$ (equiv.) & $24 \mathrm{ohm}$ & $12 \mathrm{ohm}$ \\
\hline $\mathbf{I}$ & $0.5 \mathrm{amp}$ & $1 \mathrm{amp}$ \\
\hline Va & 8.5 volt & 5 volt \\
\hline Vb & 1.5 volt & 3 volt \\
\hline Vc & 2 volt & 4 volt \\
\hline
\end{tabular}

It can be interpreted that the solution string represented by $\mathrm{W}_{1}$ is a good candidate; however, a slight change in one of its values made it the best.

\section{CONCLUSION}

This paper aims to present the framework of a new evolutionary algorithm. The basic advantage of this algorithm lies in its multi-level evaluation process. Each value is evaluated twice. First, the selected value is evaluated globally by comparing it to the other values in the solution space. Second, the selected values are evaluated locally by comparing their relative potential in affecting the objective function. The paper presents a quick overview of the philosophy, concept and optimization steps for the proposed algorithm. The algorithm is planned to be tested on several optimization benchmarking problems to evaluate its efficiency.

\section{REFERENCES}

Aguilar, J. 2004. "A General Ant Colony Model to Solve Combinatorial Optimization Problems." Revista Colombiana De Computacion, Vol. 2, No.1, 7-18. 
Christodoulou, S. 2005. "Scheduling Construction Activities Using Ant Colony Optimization." Proceedings of the Tenth International Conference on Civil, Structural, and Environmental Engineering Computing, Civil-Comp Press, Stirling, Scotland, 1-12.

Dorigo, M., and Stutzle, T. 2004. "Ant Colony Optimization." MIT Press, USA.

Elazouni, A.M., and Metwally, G. 2005. "Finance-Based Scheduling: Tool to Maximize Project Profit Using Improved Genetic Algorithms." Journal of Construction Engineering and Management, ASCE, Vol. 131, No.4, 400-412.

Elbeltagi, E., Hegazy, T., and Grierson, D. 2005. "Comparison among five evolutionary-based optimization algorithms." Advanced Engineering Informatics, Elsevier, 43-55.

El-Rayes, K., and Kandil, A. 2005. "Time-Cost-Quality Trade-Off Analysis for Highway Construction." Journal of Construction Engineering and Management, ASCE, Vol. 131, No.4, 477-486.

Feng, C.W., Liu, L., and Burns, S. 1997. "Using Genetic Algorithms To Solve Construction Time-Cost TradeOff Problems." Journal of Computing in Civil Engineering, ASCE, Vol. 11, No.3, 184-189.

Goldberg, D.E. 1989. "Genetic Algorithms in Search, Optimization, and Machine Learning." Addison Wesely Publishing Company, Inc., New York.

Haidar, A., Naoum, S., Howes, R., and Tah, J. 1999. "Genetic Algorithms Application And Testing For Equipment Selection." Journal of Construction Engineering and Management, ASCE, Vol. 125, No.1, 32-40.

Hegazy, T., and Ersahin, T. 2001. "Simplified Spreadsheet Solutions. II: Overall Schedule Optimization.” Journal of Construction Engineering and Management, ASCE, Vol. 127, No.6, 469-475.

Hegazy, T., and Wassed, N. 2001. "Simplified Spreadsheet Solutions. II: Overall Schedule Optimization.” Journal of Construction Engineering and Management, ASCE, Vol. 127, No.3, 183-191.

Khalafallah, A., and El-Rayes, K. 2006. "Minimizing Construction-Related Hazards in Airport Expansion Projects," Journal of Construction Engineering and Management, ASCE, 132(6), 562-572

Khalafallah, A., and El-Rayes, K. (2008) "Maximizing Security of Operating Airports during Expansion Projects," Journal of Construction Engineering and Management, ASCE, 134(1), 40-48

Leu, S.S., and Yang, C.H. 1999. "GA-Based Multicriteria Optimal Model For Construction Scheduling." Journal of Construction Engineering and Management, ASCE, Vol. 125, No.6, 420-427.

Lu, M. 2002. "Enhancing Project Evaluation and Review Technique Simulation through Artificial Neural Network-based Input Modeling." Journal of Construction Engineering and Management, ASCE, Vol. 128, No.5, 438-445.

Maier, H.R., Simpson, A.R., Zecchin, A.C., Foong, W. K., Phang, K.Y., Seah, H. Y., and Tan, C.L. 2003. "Ant Colony Optimization for Design of Water Distribution Systems." Journal of Water Resource Planning and Management, ASCE, Vol.129, No.3, 200-209.

Shyu, S.J., Yin, P., and Lin, B.M.T. 2004. "An Ant Colony Optimization Algorithm for the Minimum Weight Vertex Cover Problem." Annals of Operation Research, Kluwer Academic Publishers, Vol. 131, 283-304.
Solimanpur, M., Vart, P., and Shankar, R. 2004. "Ant colony optimization algorithm to the inter-cell layout problem in cellular manufacturing." European Journal Of Operational Research, Elsevier, Vol.157, 592 -606.

Winstone, Wayne L. 1993. "Operations Research Applications and algorithms." Duxbury Press, California.

Wu, S.A, and Banzhaf, W. 1998. "Introduction to Special Issues: Variable-length Representation and Noncoding Segments for Evolutionary Algorithms", Evolutionary Computation, MIT Press, Vol. 4.

Zehng, D.X.M., Ng, S., and Kumaraswamy, M.M 2005. "Applying Pareto Ranking and Niche Formation to Genetic Algorithm-Based Multiobjective Time-Cost Optimization." Journal of Construction Engineering and Management, ASCE, Vol. 131, No.1, 81-91.

\section{AUTHORS BIOGRAPHIES}

MOHAMED ABDEL-RAHEEM is a $\mathrm{PhD}$ student in the civil engineering program at the University of Central Florida. He received his bachelor degree in construction engineering from the American University in Cairo in 2003. Later, he got his master of science in civil engineering in 2006 from Cairo University, where he developed a computer-based framework for maximizing profit using evolutionary algorithms. Abdel-Raheem's research interests are artificial intelligence and its application in civil engineering, cash flow management, risk management, and contract administration.

AHMED KHALAFALLAH is currently an assistant professor at the University of Central Florida. He received a $\mathrm{PhD}$ in Civil Engineering from the University of Illinois at Urbana Champaign in 2006. Prior to that, he received a BSc degree in Civil Engineering and an MS in Construction Engineering from Cairo University in 2000 and 2002, respectively. Khalafallah has more than 9 years of research and teaching experience at a number of leading institutes including the University of Central Florida, the University of Illinois, Cairo University and Queen's University of Belfast. His research focuses on the areas of multi-objective optimization of infrastructure projects, construction risk management, utilization of intelligent information technologies to design, plan, and control infrastructure projects. 